\title{
GPPS-BJ-2019-0050
}

\section{MACHINE LEARNING UNCERTAINTY QUANTIFICATION OF SPALART-ALLMARAS TURBULENCE MODEL FOR COMPRESSORS}

\author{
Xiao He*, Fanzhou Zhao and Mehdi Vahdati \\ Department of Mechanical Engineering \\ Imperial College London \\ London SW7 2AZ, UK
}

\begin{abstract}
Turbulence models in Reynolds-Averaged Navier-Stokes simulations have a crucial effect on the compressor stability boundary. In this paper, the parametric uncertainty of the Spalart-Allmaras turbulence model on compressor flows is quantified by a metamodel-based Monte Carlo method. The model coefficients are represented by uniform distributions within intervals, and the quantities of interest include the Reynolds stress distribution, the shock front location and pressure coefficient and the separation size. An artificial neural network from machine learning is applied as the metamodel, which is tuned, trained, and tested by databases from the flow solver to achieve an error below $1 \%$ of the database range. The uncertainty of quantities of interest is determined by the range of the metamodel and the database samples from the flow solver. The sensitivity of model coefficients is quantified by calculating the gradient of quantities of interest from the metamodel. Results show that the measured data of Reynolds stress profiles in separated regions, shock front location and pressure coefficient and shock-induced separation size cannot be well captured. Crucial model coefficients on the quantities of interest are identified by a sensitivity analysis. However, re-calibration of these coefficients is contradictory in different quantities of interest and flow regimes. It indicates the need for a modified Spalart-Allmaras turbulence model form to improve the accuracy in predicting compressor flows.
\end{abstract}

\section{INTRODUCTION}

Reliability is of most importance for gas turbine compressors. A conventional approach to analyze compressor stability is to solve Reynolds-Averaged Navier-Stokes (RANS) equations with a turbulence model for closure. The
Spalart-Allmaras (SA) turbulence model [1] is one of the most popular turbulence models in compressor aerodynamics. It is a one-equation linear eddy viscosity model advantageous by its robustness and high numerical efficiency. Through the combined RANS-SA approach, understandings towards physics of surge and rotating stall were advanced: unsteady flow patterns during surge [2] and rotating stall [3,4] cycles were visualized, and vortical structures that lead to the stall inception were identified [5]. However, since the SA model is designed and calibrated in simple free shear flows and wall-bounded flows, it shows low accuracy in compressor near-stall flows [6,7] and often fails to predict the compressor stall margin.

To improve the SA model accuracy in compressor flows, several works have been carried out to modify its model form by using a combination of flow physics, intuition, and empiricism. Modifications with regard to the effects of helicity [8], adverse pressure gradient $[9,10]$, roughness and transition [11] have achieved a certain degree of success.

With the rapid development of data science, techniques such as uncertainty quantification (UQ) have brought new horizons and renewed interest in the field of turbulence modeling [12]. The purpose of conducting UQ studies on turbulence models is not to design a perfect model form that best fits the nature of turbulence; instead, it is to evaluate the level of confidence in results obtained by using an existing model that has intrinsic flaws. Origin of uncertainties in RANS turbulence models can be classified into model-form uncertainties and parametric uncertainties. The model-form uncertainties arise from assumptions that determine the mathematical form of the model (e.g., the Boussinesq approximation). The parametric uncertainties come from the model coeffcients that are calibrated by a limited number of simple flow cases. Both types of

${ }^{*}$ Corrosponding author. Email: xiao.he2014@imperial.ac.uk 
uncertainties are epistemic.

One of the pioneering UQ works on the SA turbulence model is from Godfrey and Cliff [13]. They built intrusive sensitivity equations to analyze sensitivities of SA model coefficients for predicting a subsonic flat plate boundary layer. It was concluded that the SA model coefficients $c_{v 1}, c_{b 1}, \sigma$ and $c_{w 2}$ are most influential, but no information about the uncertainty of results was obtained. Later research quantifies uncertainties through either a forward or a backward propagation framework.

The forward approach pre-assumes the probability density function (PDF) of model terms or model coefficients and then propagates it through RANS equations to obtain the PDF of the quantity of interest (QoI). An example of the forward approach is from Han and Hosder [14], where the model-form uncertainty of the SA model was investigated by multiplying the eddy viscosity by a random variable with a uniform PDF. In their study, the measured velocity profiles of the synthetic jet actuator case could be enveloped by the $95 \%$ confidence interval. Another example is from Schaefer et al. [15, 16] and Stephanopoulos et al. [17], where the non-intrusive polynomial chaos method was applied to investigate the parametric uncertainty of the SA model. The idea was to train a metamodel of RANS equations based on the response surface and then propagate the SA model coefficients with uniform PDFs through the metamodel. Their test cases included the transonic axisymmetric bump, the RAE 2822 transonic airfoil, the subsonic flat plate and the subsonic backward-facing step. It was generally found that the discrepancy between the experiment and the simulation was most evident in the separation regions, and the predicted results were sensitive to the SA model coefficients $\sigma, \kappa, c_{v 1}$, $c_{b 1}$ and $c_{w 2}$.

The backward approach firstly solves a calibration problem in a Bayesian framework, which utilizes experiment or high-fidelity simulation results to infer a proper PDF of model terms or model coefficients. The calibrated PDF is then propagated to predict the PDF of the QoI. Oliver and Moser [18] calibrated the SA model coefficients and compared the SA model with other turbulence models in a fully developed channel flow. Results showed that the calibrated SA model coefficients $\sigma$ and $\kappa$ have very similar values to their defaults, and the SA model predicted a comparable velocity profile to the $k-\varepsilon$ model and the $v^{2}-f$ model but with higher uncertainty. Cheung et al. [19] performed a similar calibration of SA model coefficients in a subsonic flat plate under different pressure gradients. Evident difference between default values and calibrated values of SA model coefficients was observed for all calibration scenarios. Papadimitriou and Papadimitriou [20] adopted an adjoint method to optimize the posterior Gaussian distribution of the SA model coefficients, and conducted similar calibration and prediction in the subsonic flat plate and the subsonic backward facing step case. The SA model was found accurate except for the separation region.

The motivation of this paper is to quantify the uncertainty of SA model coefficients on predicting flow features of compressors, and analyze the sensitivity of these coefficients to guide future model modifications. To achieve this, an
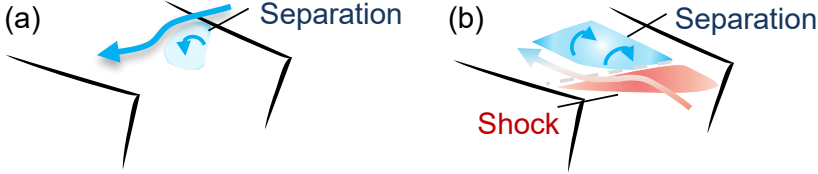

Figure 1. Illustration of compressor flow features: (a) tip leakage flow and (b) shock-boundary layer interaction (a)

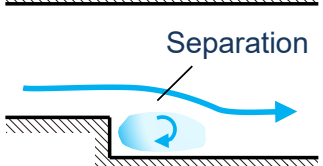

(b)

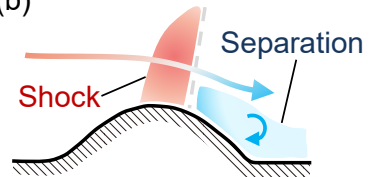

Figure 2. Illustration of (a) backward-facing step flow and (b) axisymmetric transonic bump flow

efficient forward-propagation UQ framework is proposed based on a metamodel of the flow solver. The metamodel is based on an artificial neural network from machine learning, which has presented great potential in fields of optimization, computer vision, general game playing, etc. Methodology including the flow solver, the UQ method and the metamodel will be detailed first, followed by the results and discussions on the uncertainty quantification of the Reynolds stress, the shock, and the separation.

\section{EXTRACTION OF COMPRESSOR FLOW FEATURES}

The internal flow of a modern aero compressor is featured by the interaction between the shock, the boundary layer and the tip leakage flow. The tip leakage flow illustrated in Fig. 1(a) produces significant turbulent kinetic energy and thus increases endwall viscous losses [21]. The shock structure presented in Fig. 1(b) induces boundary layer separation that enhances the blockage and the efficiency loss [22]. The tip leakage flow and the shock structure are highly interactive with each other. When compressor operates towards the stall limit, the tip leakage vortex breakdown occurs after the shock front, which leads to compressor stall [23-25]. Thus, the corner between the casing wall and the blade suction surfaces is the dominant region on determining the compressor performance [26], and special care is needed in simulations.

In this analysis, the tip leakage flow and the shock structure are studied independently by investigating simplified compressor flows, namely the backward-facing step flow and the axisymmetric transonic bump flow. The main flow physics of the step flow in Fig. 2(a) is the formation of a free shear layer, and for the bump flow in Fig. 2(b) the main flow physics is the shock-induced separation. Such an approach allows a better understanding of the turbulence modeling effect on each flow feature. Another merit of this approach is the easy access to the measured turbulent shear stress data, which is often not readily available especially for a high-speed compressor. Experimental data used in the following discussions are taken from Driver and Seegmiller [27] for the step flow and Bachalo and Johnson [28] for the bump flow. 


\section{METHODOLOGY}

\section{The Spalart-Allmaras Turbulence Model}

The SA turbulence model [1] is a linear eddy viscosity model based on the Boussinesq assumption as Eqn. (1):

$$
\tau_{i j}=2 \mu_{t} S_{i j}
$$

where the turbulent shear stress $\tau_{i j}$ is linearly corelated with the mean strain rate $S_{i j}$, and the turbulent viscosity $\mu_{t}$ is solved by Eqn. (2):

$$
\mu_{t}=\rho \hat{v} f_{v 1}
$$

The modified turbulent viscosity $\hat{v}$ is solved by the transport equation shown in Eqn. (3):

$$
\begin{aligned}
\frac{\partial \hat{v}}{\partial t}+u_{j} \frac{\partial \hat{v}}{\partial x_{j}} & =c_{b 1}\left(1-f_{t 2}\right) \hat{S} \hat{v}-\left[c_{w 1} f_{w}-\frac{c_{b 1}}{\kappa^{2}} f_{t 2}\right]\left(\frac{\hat{v}}{d}\right)^{2} \\
& +\frac{1}{\sigma}\left[\frac{\partial}{\partial x_{j}}\left((v+\hat{v}) \frac{\partial \hat{v}}{\partial x_{j}}\right)+c_{b 2} \frac{\partial \hat{v}}{\partial x_{i}} \frac{\partial \hat{v}}{\partial x_{i}}\right]
\end{aligned}
$$

where the modified strain rate $\hat{S}$ is defined as Eqn. (4):

$$
\hat{S}=\Omega+\frac{\hat{v}}{\kappa^{2} d^{2}} f_{v 2}
$$

and additional variables is calculated in Eqn. (5) using coefficients summarized in Table 1. Among these equations, $\rho$ is density, $\Omega$ is vorticity magnitude, and $d$ is wall distance.

$$
\begin{aligned}
c_{w 1} & =\frac{c_{b 1}}{\kappa^{2}}+\frac{1+c_{b 2}}{\sigma} & f_{v 1} & =\frac{\chi^{3}}{\chi^{3}+c_{v 1}^{3}} \quad \chi=\frac{\hat{v}}{v} \\
f_{v 2} & =1-\frac{\chi}{1+\chi f_{v 1}} & f_{w} & =g\left[\frac{1+c_{w 3}^{6}}{g^{6}+c_{w 3}^{6}}\right]^{1 / 6} \\
g & =r+c_{w 2}\left(r^{6}-r\right) & r & =\min \left[\frac{\hat{v}}{\hat{S} \kappa^{2} d^{2}}, 10\right] \\
f_{t 2} & =c_{t 3} \exp \left(-c_{t 4} \chi^{2}\right) & &
\end{aligned}
$$

In this paper, all the SA model coefficients are investigated except for transition-related terms $c_{t 3}$ and $c_{t 4}$. The physical relevance and the investigated interval of the SA model coefficients are shown in Table 1. The intervals of coefficients were verified by Spalart as reported in Schaefer et al. [15]. Most of the intervals are determined from the calibration process concerning free shear flows and simple wall-attached flows [1].

\section{Flow Solver, Validation and Verification}

The in-house computational fluid dynamics (CFD) solver AU3D solves the three-dimensional, viscous and compressible RANS equations based on a cell-vertex finite volume scheme.
Table 1. Intervals of Spalart-Allmaras turbulence model coefficients

\begin{tabular}{cllll}
\hline Coef. & Physical relevance & Min. & Dflt. & Max. \\
\hline$\sigma$ & diffusion coefficient & 0.600 & 0.667 & 1.000 \\
$\kappa$ & log law slope & 0.38 & 0.41 & 0.42 \\
$c_{v 1}$ & log law intercept & 6.9 & 7.1 & 7.3 \\
$c_{b 1}$ & production coefficient & 0.128 & 0.1355 & 0.137 \\
$c_{b 2}$ & conserve $v_{t}^{1+c_{b 2}}$ & 0.609 & 0.622 & 0.688 \\
$c_{w 2}$ & calibrate skin friction & 0.055 & 0.300 & 0.353 \\
$c_{w 3}$ & speed up destruction & 1.75 & 2.00 & 2.50 \\
$c_{t 3}$ & calibrate transition & - & 1.2 & - \\
$c_{t 4}$ & calibrate transition & - & 0.5 & - \\
\hline
\end{tabular}

(a)

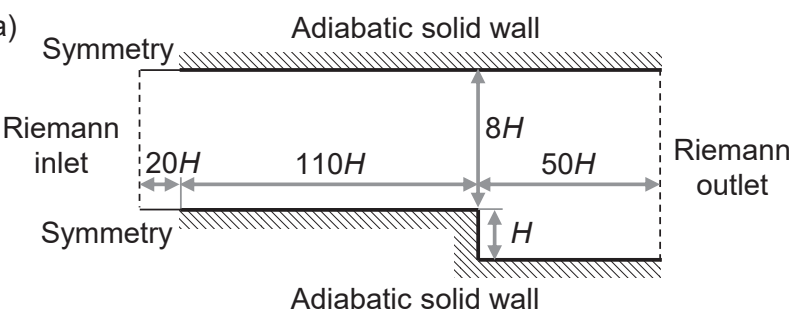

(b)

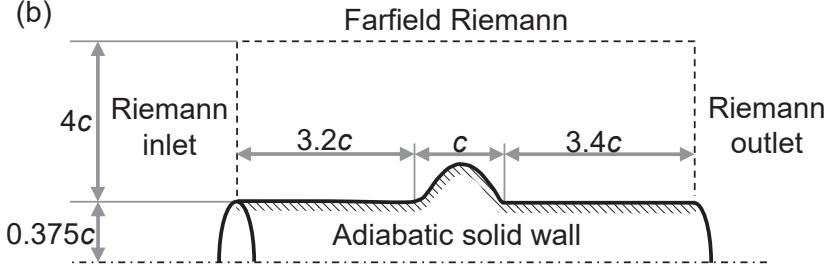

Figure 3. Geometry and boundary conditions for (a) backward-facing step flow and (b) axisymmetric transonic bump flow

The Jameson-Schmidt-Turkel scheme with the Roe's flux vector difference splitting method is applied to solve numerical fluxes, and second-order accuracy is achieved in both time and space. The solver has been used in turbomachinery flows for more than 20 years. More details of the solver can be found in Sayma et al. [29].

Boundary conditions used for the step case and the bump case are presented in Fig. 3. Mesh size and operating conditions are summarized in Table 2. The meshes in use are the second finest provided by the Turbulence Modeling Resouce website ${ }^{1}$, which were verified by AU3D to produce mesh independent results.

Results from AU3D are compared with experimental data and CFL3D simulations in Fig. 4, where the surface pressure coefficient is plotted against the axial distance normalized by the step height $(H)$ or the bump chord length $(c)$. The step is located at $x / H=0$, and the bump starts from $x / c=$ 0 . It is illustrated that the AU3D results overlap with that from CFL3D, which verifies the implementation of the original Spalart-Allmaras (OSA) model. An evident difference

\footnotetext{
${ }^{1}$ Data retrieved in Nov. 2018 from https://turbmodels.larc.nasa.gov/
} 
Table 2. Mesh size and operating conditions

\begin{tabular}{lllll}
\hline Case & Mesh Size & Re & Ma & $T(\mathrm{~K})$ \\
\hline Step & $2 \times 354 \times 257$ & $3.6 \times 10^{4}$ & 0.15 & 298.33 \\
& $2 \times 514 \times 449$ & & & \\
Bump & $2 \times 721 \times 321$ & $2.763 \times 10^{6}$ & 0.875 & 300 \\
\hline
\end{tabular}
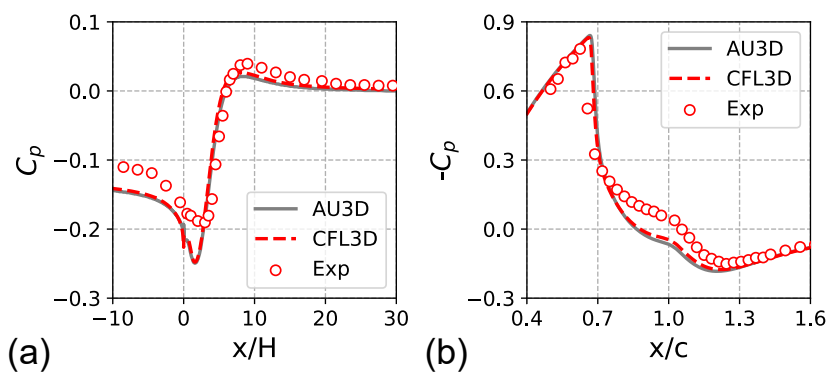

Figure 4. Validation and verification on surface pressure coefficient of (a) the step case and (b) the bump case

between experiments and both simulations is also captured, indicating the deficiency of the OSA model.

\section{Uncertainty Quantification Method}

Conventional UQ methods like Monte Carlo require loads of numerical resources to approximate the solution with high accuracy. In this paper, the parametric uncertainty of SA model coefficients is investigated through a metamodel-based Monte Carlo approach so that the required numerical resources can be alleviated.

Firstly, the Latin Hypercube sampling method [30] is applied to generate five independent databases of SA model coefficient sets within the interval in Table 1. This Design of Experiment (DOE) method is able to distribute the samples as uniformly as possible in the investigated space. The database size is $16,32,64,128$ and 256 respectively, which will be referred to as $\mathrm{db} 1$ to $\mathrm{db} 5$ in following discussions. By calculating the Euclidean distance between arbitrary two samples from different databases, it has been verified that all of the five databases are very different from each other. The total amount of numerical resources for running these databases by AU3D in both the step and the bump case are about 140,000 core hour.

The generated databases are then applied to train and test a metamodel of CFD. The metamodel is a reduced-order model that directly links the input variables with quantities of interest (QoIs) without solving RANS equations:

$$
\hat{\boldsymbol{q}} \approx \boldsymbol{q} \equiv \boldsymbol{M}(\boldsymbol{x})
$$

where $\boldsymbol{x}$ is the vector of input variables, $\boldsymbol{M}(\cdot)$ denotes the metamodel, $\hat{\boldsymbol{q}}$ and $\boldsymbol{q}$ are the vector of QoIs from CFD and the metamodel, respectively.

Finally, the uncertainty of QoIs can be quantified by the maximum and the minimum values predicted by the

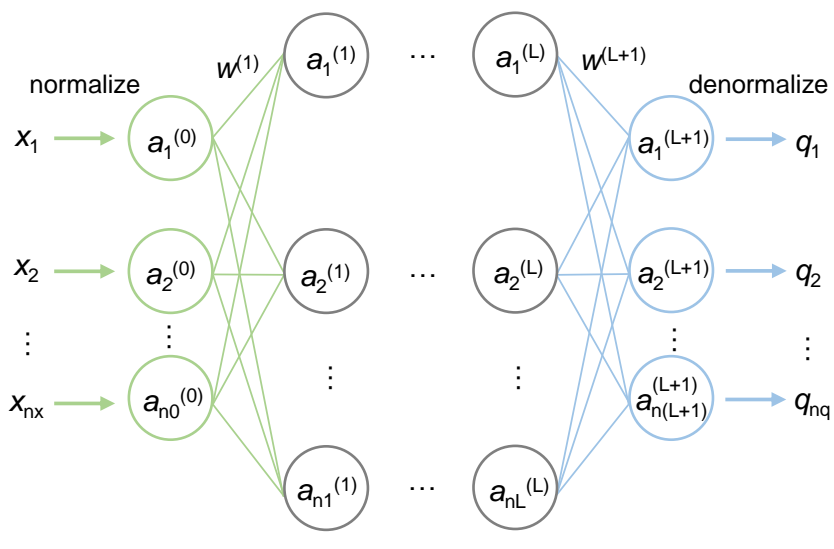

Input layer Hidden layers Output layer

Figure 5. The architecture of artificial neural network

metamodel. The PDF of QoIs can be obtained when the PDF of input variables is pre-assumed, to which the uniform distribution is used in this work. The sensitivity of input variables on predicting QoIs can be quantified by the normalized gradient calculated from the metamodel, as defined in Eqn. (7):

$$
\widetilde{\nabla q_{i}}=\frac{\nabla M_{i}}{\left\|\nabla M_{i}\right\|}
$$

\section{Metamodel based on Machine Learning}

In this study, the artificial neural network (ANN) from the field of machine learning is applied to construct a metamodel of the CFD solver. The mathmatical form and the working principle of the ANN are detailed as follows.

Artificial Neural Network Representation The ANN is composed of several levels of artificial neurons, as illustrated in Fig.5. The input variables, the intermediate values, and the QoIs are stored in the input layer, hidden layers and the output layer. In this case, the SA model coefficients are fed in the input layer, and the QoIs including the Reynolds stress profiles, the shock front location and pressure coefficient and the separation size are imposed at the output layer, as denoted by Eqn. (8):

$$
\begin{aligned}
& \boldsymbol{x}= {\left[\sigma, \kappa, c_{v 1}, c_{b 1}, c_{b 2}, c_{w 2}, c_{w 3}\right]^{T} } \\
& \boldsymbol{q}= \begin{cases}{\left[\tau\left(y_{1}\right), \tau\left(y_{2}\right), \cdots, \tau\left(y_{j}\right)\right]^{T},} & \text { (Reynolds stress) } \\
{\left[C_{p s f}, x_{s f}\right]^{T},} & \text { (shock) } \\
{\left[L_{s}\right]^{T},} & \text { (separation) }\end{cases}
\end{aligned}
$$

where the shock front is defined as the location with the lowest wall pressure, the separation size of the step case is defined between the step location and the reattachment point, and the separation size of the bump case is defined between the separation and the reattachment points. Both the separation and the reattachment points are determined by the zero skin friction locations. 
Prediction by Forward Propagation The ANN predicts the QoIs by the following steps. Firstly, the SA model coefficients are normalized by their maximum and minimum values presented in Table 1:

$$
a_{i}^{(0)}=\frac{x_{i}^{(j)}-\min x_{i}}{\max x_{i}-\min x_{i}}
$$

where $\boldsymbol{x}^{(j)}$ is the $j^{\text {th }}$ sample of the test database $\left\{\left(\boldsymbol{x}^{(j)}, \hat{\boldsymbol{q}}^{(j)}\right) \mid 1 \leq j \leq m_{t e}\right\}$. The values at the $l^{\text {th }}$ layer are then propagated to the $(l+1)^{\text {th }}$ layer using Eqn. (10):

$$
\boldsymbol{a}^{(l+1)}=g\left(\boldsymbol{z}^{(l+1)}\right)=g\left(\boldsymbol{w}^{(l+1)} \boldsymbol{a}^{(l)}+\boldsymbol{b}^{(l+1)}\right)
$$

where $\boldsymbol{w}$ is the weight matrix, $\boldsymbol{b}$ is the bias vector, and $g(\cdot)$ is the activation function to which the non-linear sigmoid function is applied. Finally, values at the output layer are denormalized to produce the QoIs. The QoIs are normalized by Eqn. (11):

$$
a_{i}^{(L+1)}=\frac{1}{\omega} \frac{q_{i}^{(j)}-\min _{1 \leq k \leq m_{t r}} \hat{q}_{i}^{(k)}}{\max _{1 \leq k \leq m_{t r}} \hat{q}_{i}^{(k)}-\min _{1 \leq k \leq m_{t r}} \hat{q}_{i}^{(k)}}+\frac{\omega-1}{2 \omega}
$$

where $\omega$ is the relaxation factor that scales QoIs to the range of $0.5 \pm 0.5 \omega^{-1}$, and $\hat{\boldsymbol{q}}^{(k)}$ is the $k^{\text {th }}$ sample of the training database $\left\{\left(\boldsymbol{x}^{(k)}, \hat{\boldsymbol{q}}^{(k)}\right) \mid 1 \leq k \leq m_{t r}\right\}$. The prediction accuracy of the ANN can be measured by the relative error defined by Eqn. (12):

$$
\varepsilon=\frac{1}{m} \sum_{j=1}^{m} \sum_{i=1}^{n q} \frac{\left|\hat{q}_{i}^{(j)}-q_{i}^{(j)}\right|}{\max _{1 \leq k \leq m_{t r}} \hat{q}_{i}^{(k)}-\min _{1 \leq k \leq m_{t r}} \hat{q}_{i}^{(k)}} \times 100 \%
$$

Learning with Backpropagation The accuracy of the ANN depends on the network parameters $\boldsymbol{w}^{(l)}$ and $\boldsymbol{b}^{(l)}$, which are initialized randomly before training in this study. Calibration of those parameters, or training of the ANN, is achieved by solving an optimization problem that minimizes the cost function $J$ defined by Eqn. (13):

$$
\begin{aligned}
J\left(w_{i, k}^{(l)}, b_{i}^{(l)}\right)= & \frac{1}{2 m} \sum_{j=1}^{m} \sum_{i=1}^{n_{L+1}}\left(q_{i}^{(j)}-\hat{q}_{i}^{(j)}\right)^{2} \\
& +\frac{\lambda}{2 m} \sum_{l=1}^{L+1} \sum_{i=1}^{n_{l}} \sum_{k=1}^{n_{l-1}}\left(w_{i, k}^{(l)}\right)^{2}
\end{aligned}
$$

where $\lambda$ is a regularization factor that penalizes large-valued weights in order to avoid overfitting. In this paper, the gradient-based Broyden-Fletcher-Goldfarb-Shanno (BFGS) optimizer [31] is applied. The gradient of the cost function

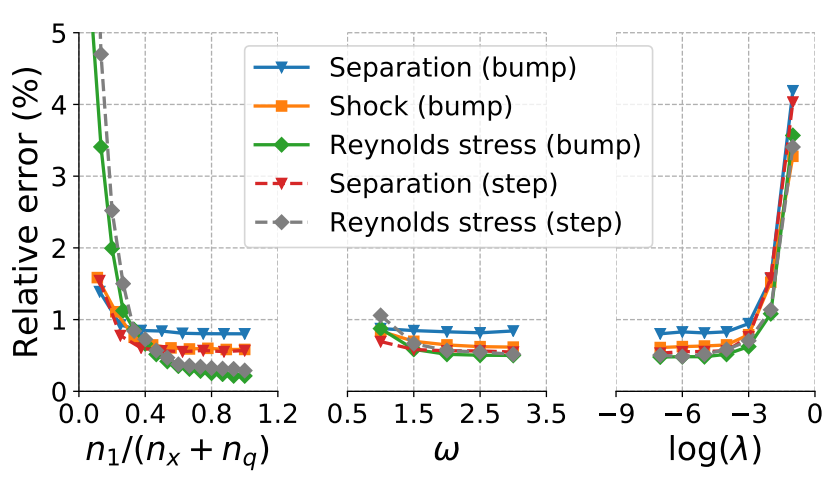

Figure 6. Parametric study of hidden layer neuron number, relaxation factor and regularization factor (training data: db5; cross-validation data: the union of $\mathrm{db} 1, \mathrm{db} 2$ and $\mathrm{db} 3$ )

is calculated by the backpropagation algorithm as follows:

$$
\begin{aligned}
\frac{\partial J}{\partial \boldsymbol{w}^{(l)}} & =\frac{1}{m} \sum_{j=1}^{m} \boldsymbol{\delta}^{(l)} \cdot\left(\boldsymbol{a}^{(l-1)}\right)^{T}+\frac{\lambda}{m} \boldsymbol{w}^{(l)} \\
\frac{\partial J}{\partial \boldsymbol{b}^{(l)}} & =\frac{1}{m} \sum_{j=1}^{m} \boldsymbol{\delta}^{(l)}
\end{aligned}
$$

with the $\boldsymbol{\delta}^{(l)}$ defined as follows:

$$
\boldsymbol{\delta}^{(l)}= \begin{cases}\left(\boldsymbol{a}^{(L+1)}-\hat{\boldsymbol{a}}^{(L+1)}\right) \cdot * g^{\prime}\left(\boldsymbol{z}^{(L+1)}\right), & l=L+1 \\ \left(\boldsymbol{w}^{(l+1)}\right)^{T} \boldsymbol{\delta}^{(l+1)} \cdot * g^{\prime}\left(\boldsymbol{z}^{(l)}\right), & 1 \leq l \leq L\end{cases}
$$

It should be noted that the gradient-based optimization methods may lead to a local optimum result rather than the global optimum. To reduce the chance of falling into a local optimum, 16 ANNs with differernt initial conditions are trained parallelly, and the best ANN is chosed.

Hyperparameter Study In addition to the standard parameters that can be learned from the training database, there are some hyperparameters that need to be determined before the learning process, namely the number of hidden layers $(L)$, the number of artificial neurons in each hidden layer $\left(n_{L}\right)$, the relaxation factor $(\omega)$ and the regularization factor $(\lambda)$.

Since the simple ANN architecture with only one hidden layer achieves a satisfactory accuracy, it is adopted throughout the paper (i.e., $L=1$ ). A parametric study of $n_{1}, \omega$, and $\lambda$ has been conducted by changing one hyperparameter at a time while keeping the others the same with the baseline setting, which is $n_{1}=0.5\left(n_{x}+n_{q}\right), \omega=2$ and $\lambda=10^{-4}$. The prediction errors of ANNs in various QoIs are plotted against each hyperparameter in Fig. 6. It is observed that the hyperparameter settings of $n_{1} \geq 0.5\left(n_{x}+n_{q}\right), \omega \geq 2$ and $\lambda \leq 10^{-4}$ lead to a high-accuracy ANN model with the relative errors of all QoIs below 1\%. Thus, the baseline setting is adopted throughout the paper.

Training Data Independence Study The accuracy of the ANN generally improves when feeding in a larger 


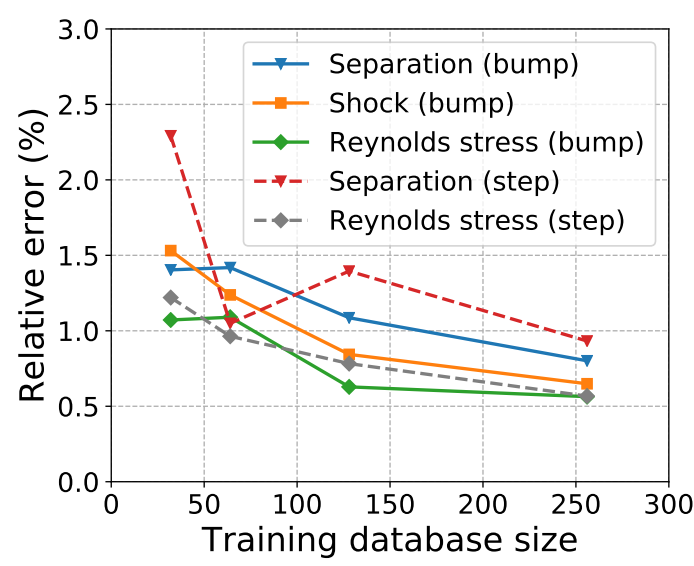

Figure 7. Training data independence study

training database. A training data independence study has been performed by using different combinations of databases to train and test the ANN. In this case, the training and test database are $\mathrm{db}\{i\}$ and $\mathrm{db}\{i-1\}$ respectively, so that the ratio of training database size to test database size can be kept the same as 2. The prediction errors of ANNs in various QoIs are plotted against the training database size in Fig. 7 . It is illustrated that the training database size of 256 surpasses the critical value where the relative error has the highest rate to decrease. Thus, the database with 256 samples has been chosen to train ANNs in the following discussions.

\section{RESULTS}

\section{Uncertainty on Reynolds Stress Prediction}

The Reynolds stress profiles at different sections of the step case are illustrated in Fig. 8. In this plot, the light blue regions represent all CFD results from the SA model, the dark blue curves show the CFD results from the original SA model, the dashed grey curves denote the minimum and the maximum Reynolds stress bounds from the ANN, and the red circles represent the experimental data. It is shown that the Reynolds stress profile has the largest uncertainty when the flow just separates from the boundary at Sec. I. As the flow develops to Sec. II and III, the uncertainty of Reynolds stress decreases, and the peaks of the Reynolds stress profiles from the SA model shift upward compared to the experimental data. Consequently, the experimental results cannot be fully enveloped by the SA model results in the separation region. When the flow is reattached at further downstream locations at Sec. IV, the deficiency of the SA model decreases, and the results from SA models match well with the experiment.

The Reynolds stress profiles at different sections of the bump case are illustrated in Fig. 9, which is plotted in a similar fashion as that of the step case. Similar to the observation of the step case, the uncertainty of predicted Reynolds stress and the discrepancy between predictions and the experiment are much higher in the separated region from Sec. II to Sec. VI than elsewhere. Moreover, the peak values of the Reynolds stress profiles from SA models are smaller than the experiment, indicating the inadequate turbulence production of the SA model.
To further evaluate the effect of different SA model coefficients on predicting the Reynolds stress, sensitivity analyses of the step case and the bump case are performed in Fig. 10 and Fig. 11. The mean value and the standard deviation of the normalized gradient of Reynolds stress over the investigated coefficient intervals are denoted by the height of bars and the length of error bars, respectively.

In general, the SA model coefficients $\sigma, \kappa, c_{v 1}, c_{b 1}$ and $c_{w 2}$ are found significant, while $c_{b 2}$ and $c_{w 3}$ have negligible effects. The correlation between the crucial coefficients and the Reynolds stress is determined by the wall distance, the local strain rate and the surrounding field of turbulent viscosity. At near-wall locations, the destruction term dominates the turbulent viscosity budget, and an increase in $\sigma$ will reduce the destruction and thus raise the Reynolds stress. At far-wall locations, the destruction term becomes negligible, and a decrease in $\sigma$ will increase the diffusion and thus increase the Reynolds stress. To match with the measured downward-shifted peak of Reynolds stress profiles, a higher $\sigma$ value is required. The signs of gradients with respect to other source-related coefficients, namely $\kappa, c_{v 1}, c_{b 1}$ and $c_{w 2}$, differ in locations and cases. No general conclusion on the modification of coefficients can be made at this stage.

\section{Uncertainty on Shock Prediction}

The shock of the bump case can be visualized in the wall pressure coefficient plot, as presented in Fig. 12. The shock front is illustrated in the red dashed circle followed by a drastic pressure increase due to shock compression. The uncertainties of predicted wall pressure coefficient are most evident at regions of the shock and the shock-induced separtion, where the axial location $x / c$ ranges from 0.6 to 1.2 . However, the measured wall pressure coefficient at these regions cannot be fully enveloped by predictions.

Uncertainty of SA model coefficients on predicting the shock front location and pressure coefficient is illustrated in Fig. 13. In the main plot, the shock front location is plotted against the negative of the shock front pressure coefficient. The plus signs represent all CFD results from the SA model, the triangle shows the CFD result from the original SA model, the red circle denotes the experimental result, and the contour illustrates the sample frequency from ANN. In the top and the right plots, the histogram represents the distribution of samples, and the curves are PDFs fitted by the kernel density estimation method [32] based on Gaussian kernels. It is observed that the SA model predicts a more downstream shock front location and a much lower pressure coefficient at the shock front, indicating the deficiency of SA model in shock capturing. Another observation is the clear correlation between the shock front location and the shock front pressure coefficient. This is because the pre-shock flow will experience an extended supersonic expansion process that further decreases the pressure coefficient if the shock front location is delayed.

Sensitivity analysis of shock prediction is given in Fig. 14. It is created in a similar fashion as that of Reynolds stress prediction. Similar to the observation in the sensitivity analyses of Reynolds stress, the SA model coefficients $\sigma, \kappa$, 


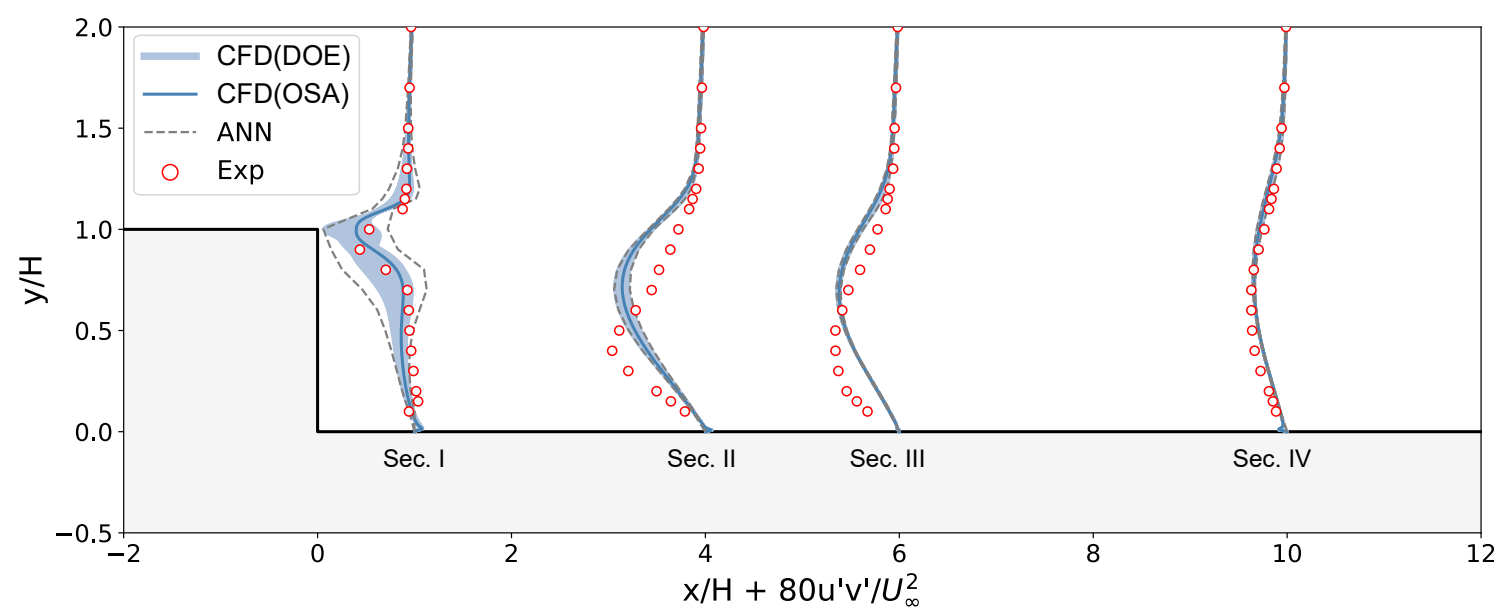

Figure 8. Uncertainty of predicted Reynolds stress profiles of the step case

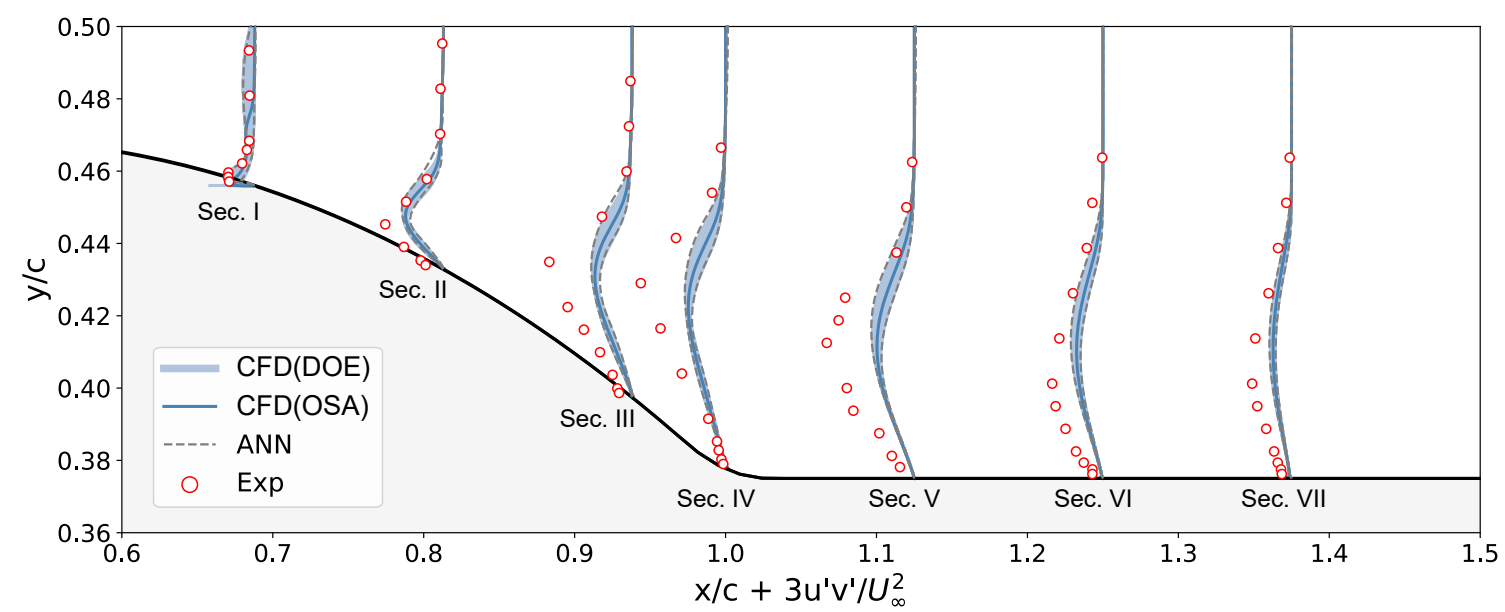

Figure 9. Uncertainty of predicted Reynolds stress profiles of the bump case

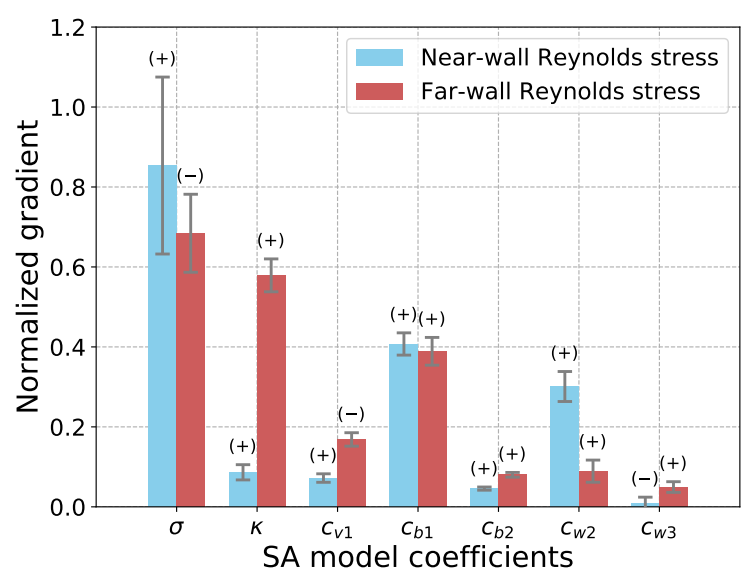

Figure 10. Sensitivity of Sparlart-Allmaras turbulence model coefficients on predicting Reynolds stress of the step case $(x / H=4$, near-wall $y / H=0.60, y^{+} \approx 540$; far-wall $y / H=1.15, y^{+} \approx 1040$ )

$c_{v 1}, c_{b 1}$ and $c_{w 2}$ are found significant, while $c_{b 2}$ and $c_{w 3}$ are negligible. Because the location and the pressure coefficient of the shock front are physically correlated with each other, they have a similar trend with the SA model coefficients. A decrease in the crucial coefficients will reduce the discrepancy

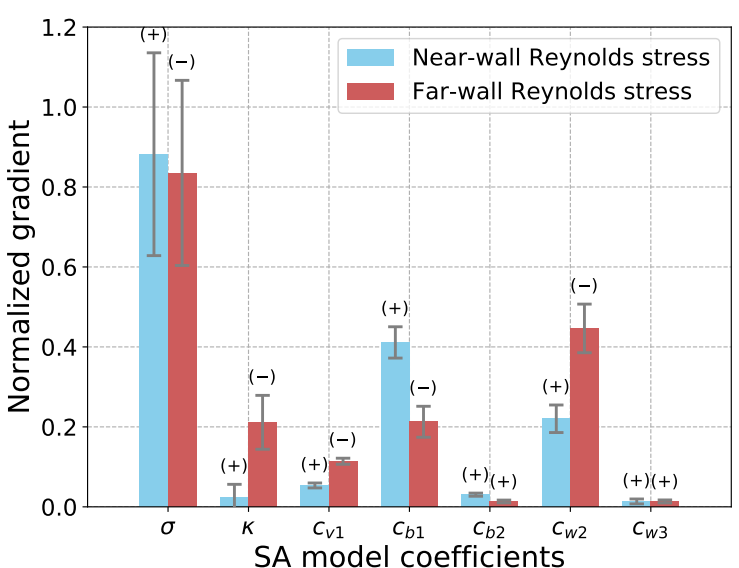

Figure 11. Sensitivity of Sparlart-Allmaras turbulence model coefficients on predicting Reynolds stress of the bump case $\left(x / c=1\right.$, near-wall $y / c=0.4012, y^{+} \approx 910 ; \quad$ far-wall $y / c=0.4387, y^{+} \approx 2220$ )

in the shock front location and pressure coefficient. However, the decrease of $\sigma$ will increase the discrepancy in Reynolds stress. The trade-off between Reynolds stress and shock predictions indicates the deficiency of the SA model form. 


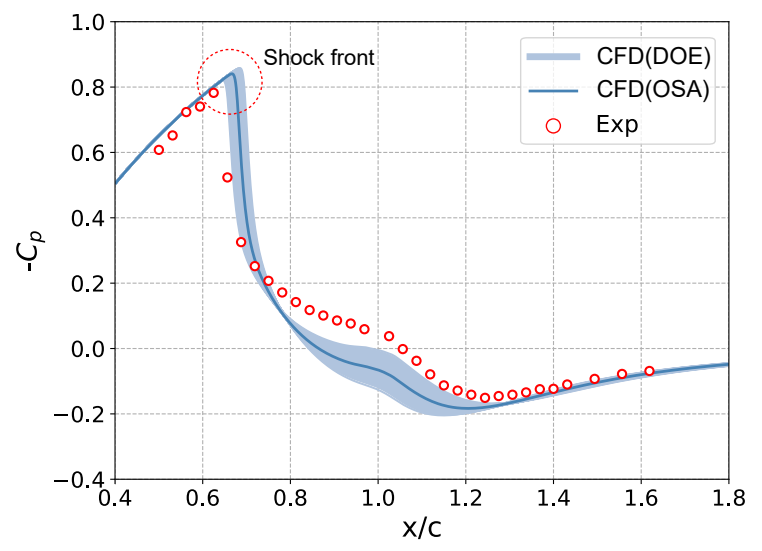

Figure 12. Uncertainty of surface pressure coefficient of the bump case
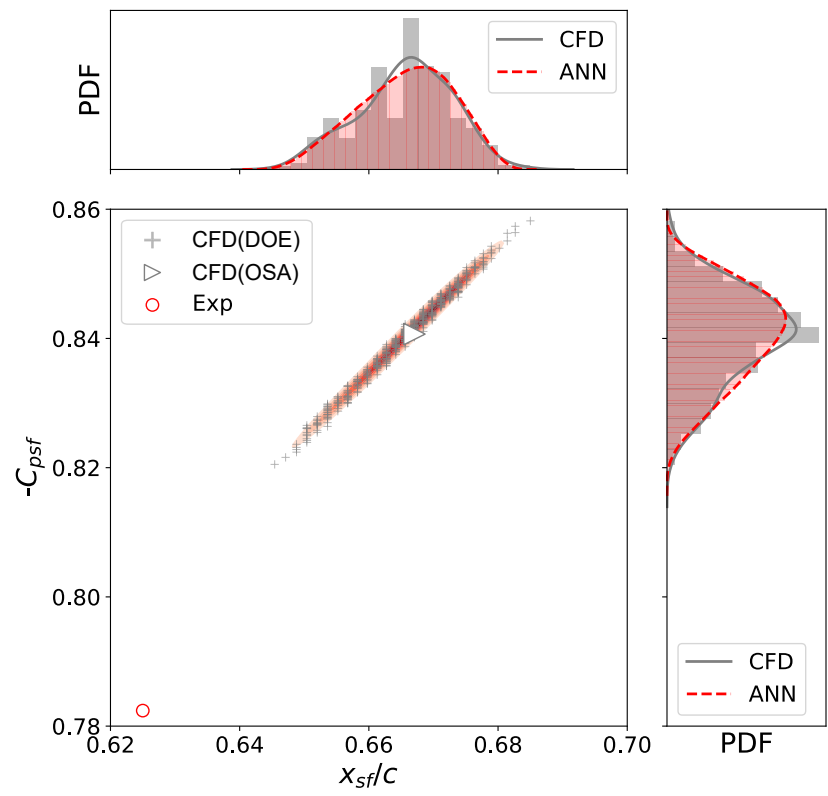

Figure 13. Uncertainty and probability density functions of predicted shock front location $\left(x_{s f} / c\right)$ and shock front pressure coefficient $\left(-C_{p s f}\right)$ in the bump case (scatters: samples from experiment and CFD; contours: sample frequency of ANN)

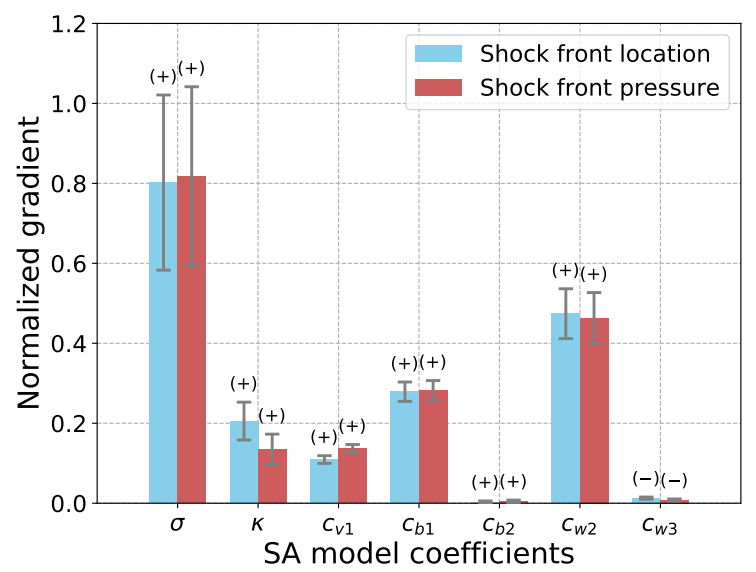

Figure 14. Sensitivity of Sparlart-Allmaras turbulence model coefficients on predicting the shock front location $\left(x_{s f} / c\right)$ and the negative of shock front pressure coefficient $\left(-C_{p s f}\right)$ of the bump case

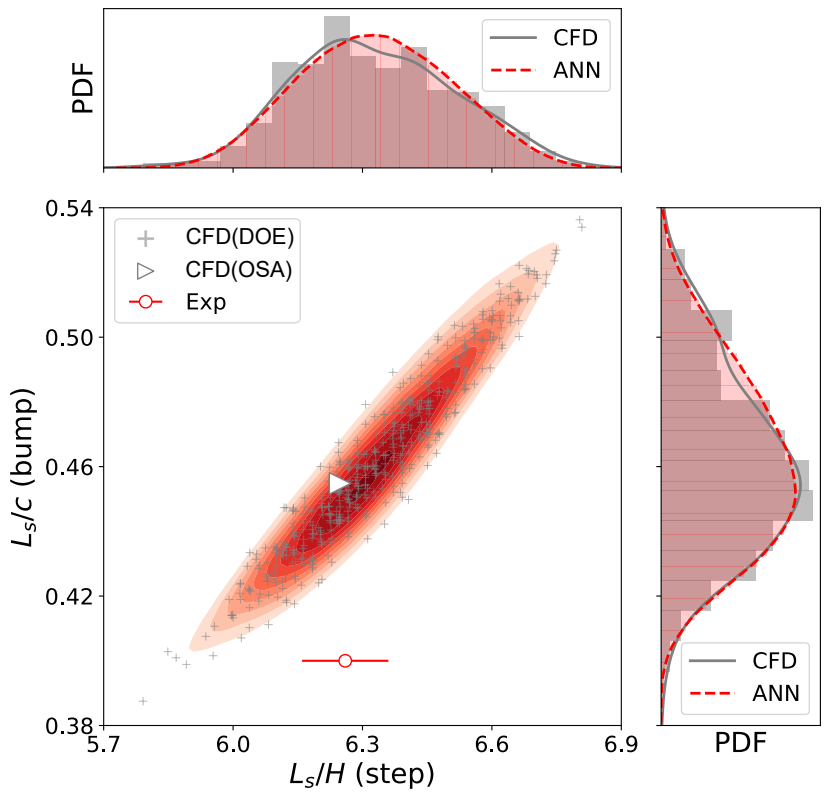

Figure 15. Uncertainty and probability density functions of predicted separation size in the step $\left(L_{S} / c\right)$ and the bump $\left(L_{S} / H\right)$ case (scatters: samples from experiment and CFD; contours: sample frequency of ANN)

\section{Uncertainty on Separation Prediction}

Uncertainty of SA model coefficients on predicting the separation size of the step case and the bump case is illustrated in Fig. 15, which is plotted in a similar fashion to that of shock prediction. It is illustrated that the measured separation size of the step case is close to the peak of the PDF from CFD and ANN, which indicates good accuracy of the SA model. However, the SA model predicts a much larger separation size in the bump case. Therefore, the experiment dot falls out of the range of SA model results in the main plot. Considering the shock-induced separation size is negatively correlated with the shock front location, the discrepancy in shock-induced separation size will be even larger if the SA model predicts the shock front location accurately.

Sensitivity analysis of the separation prediction is presented in Fig. 16. Regardless of the cause of separation, the separation size of the step case and the bump case has a similar trend with the SA model coefficients. The SA model coefficients $\sigma, \kappa, c_{b 1}, c_{w 2}$ and $c_{w 3}$ are found significant, while $c_{v 1}$ and $c_{b 2}$ are negligible. To reduce the discrepancy between experimental and the SA model results, increased values in $\sigma, \kappa, c_{b 1}$ and $c_{w 2}$ are expected, which contradicts with the re-calibration result from the shock prediction. This parodox again illustrates the deficiency of the SA model form. The coefficient $\sigma$ appears in both the diffusion term and the destruction term. An increased $\sigma$ will lead to a reduction in both terms, which has an opposite effect on the separation size. From Fig. 16, it is inferred that the $\sigma$ value in the destruction term is the dominating mechanism, which highlights the significance of the source term. 


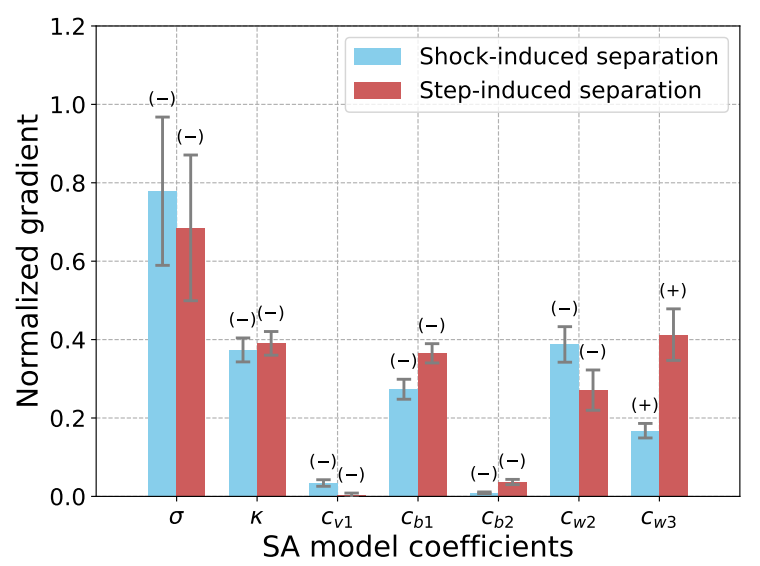

Figure 16. Sensitivity of Sparlart-Allmaras turbulence model coefficients on predicting separation size

\section{CONCLUSIONS AND FUTURE WORKS}

In this paper, a machine learning assisted uncertainty quantification framework is presented. Using the established framework, the parametric uncertainty of the SA model coefficients on predicting compressor flows has been investigated.

The SA model with different sets of model coefficients successfully predicts Reynolds stress profiles of wall-attached flows and the step-induced separation size. However, predictions of Reynolds stress profiles of separated flows, shock front location and pressure coefficient, and shock-induced separation size show discrepancy compared to the measurement. The uncertainty of predicted results is larger in the separated region than elsewhere in the domain. Such results indicate the limitation of the SA model on predicting separated flows.

Shared sensitive coefficients on predicting Reynolds stress, shock and separation are $\sigma, \kappa, c_{b 1}$ and $c_{w 2}$. The coefficient $\sigma$ occurs in both the diffusion term and the destruction term. The values in both terms affect the shape of Reynolds stress profiles, but the value in the destruction term is crucial for separation size. The coefficient $\kappa$ corrects the destruction term concerning the log-law. However, its physical meaning becomes vague in separated flow. The coefficient $c_{b 1}$ and $c_{w 2}$ controls the magnitude of the production term and the destruction term, respectively. Such results highlight the significance of the source term.

Re-calibration of model coefficients to improve the accuracy of the SA model is only plausible when considering a limited number of QoIs under certain flow regimes. When considering multiple QoIs across different flow regimes, the re-calibration will lead to contradictory results. In this study, the measured Reynolds stress and separation size of the bump flow favors an increased value of $\sigma$, but the measured shock front location and pressure coefficient indicates a lower value of $\sigma$. It implies the deficiency of the SA model form on predicting complex flows, such as the internal flow of a compressor. Modification of the SA model form is required in future research.

\section{ACKNOWLEDGMENT}

Xiao He greatly acknowledges the Imperial College President PhD Scholarship for funding this research.

$\begin{array}{ll}\text { NOMENCLATURE } \\ b & \text { bias term } \\ c & \text { bump chord length } \\ C_{p} & \text { pressure coefficient } \\ C_{p s f} & \text { shock front pressure coefficient } \\ d & \text { wall distance } \\ g(\cdot) & \text { activation function } \\ H & \text { step height } \\ J(\cdot) & \text { cost function } \\ L & \text { number of hidden layers } \\ L_{s} & \text { separation size } \\ \mathrm{M} & \text { Mach number } \\ m & \text { number of database samples } \\ \boldsymbol{M}(\cdot) & \text { metamodel } \\ n_{l} & \text { number of neurons in } l^{\text {th }} \text { hidden layer } \\ \boldsymbol{q} & \text { vector of quantaty of interest } \\ \text { Re } & \text { Reynolds number } \\ S_{i j} & \text { mean strain rate } \\ w & \text { weight term } \\ \boldsymbol{x} & \text { vector of input variables } \\ x_{s f} & \text { shock front location } \\ \varepsilon & \text { relative error } \\ \lambda & \text { regularization factor } \\ \mu_{t} & \text { dynamic turbulent viscosity } \\ \rho & \text { density } \\ \tau & \text { turbulent shear stress } \\ \hat{v} & \text { modified kinetic turbulent viscosity } \\ \omega & \text { relaxation factor } \\ \Omega & \text { vorticity } \\ & \end{array}$

\section{REFERENCES}

[1] Spalart, P., and Allmaras, S., 1994. "A one-equation turbulence model for aerodynamic flows". Rech. Aerospatiale, 1, pp. 5-21.

[2] Zhao, F., Dodds, J., and Vahdati, M., 2018. "Poststall behavior of a multistage high speed compressor at off-design conditions". ASME J. Turbomach., 140(12), p. 121002.

[3] Dodds, J., and Vahdati, M., 2015. "Rotating stall observations in a high speed compressor-part II: Numerical study". ASME J. Turbomach., 137(5), p. 051003.

[4] Gourdain, N., Burguburu, S., Leboeuf, F., and Michon, G. J., 2010. "Simulation of rotating stall in a whole stage of an axial compressor". Comput. Fluids, 39(9), pp. 1644-1655.

[5] Pullan, G., Young, A. M., Day, I. J., Greitzer, E. M., and Spakovszky, Z. S., 2015. "Origins and structure of spike-type rotating stall”. ASME J. Turbomach., 137(5), p. 051007.

[6] Denton, J. D., 2010. "Some limitations of turbomachinery CFD". ASME Paper No. GT2010-22540. 
[7] Day, I. J., 2016. "Stall, surge, and 75 years of research". ASME J. Turbomach., 138(1), p. 011001.

[8] Liu, Y., Lu, L., Fang, L., and Gao, F., 2011. "Modification of Spalart-Allmaras model with consideration of turbulence energy backscatter using velocity helicity". Phys. Lett. A, 375(24), pp. 2377-2381.

[9] Lee, K. B., Wilson, M., and Vahdati, M., 2018. "Validation of a numerical model for predicting stalled flows in a low-speed fan-part I: Modification of Spalart-Allmaras turbulence model". ASME J. Turbomach., 140(5), p. 051008.

[10] Lee, K. B., Dodds, J., Wilson, M., and Vahdati, M., 2018. "Validation of a numerical model for predicting stalled flows in a low-speed fan-part II: Unsteady analysis". ASME J. Turbomach., 140(5), p. 051009.

[11] Oriji, U. R., and Tucker, P. G., 2014. "Modular turbulence modeling applied to an engine intake". ASME J. Turbomach., 136(5), p. 051004.

[12] Duraisamy, K., Iaccarino, G., and Xiao, H., 2019. "Turbulence modeling in the age of data". Annu. Rev. Fluid Mech., 51, pp. 357-377.

[13] Godfreyand, A., and Cliff, E., 2001. "Sensitivity equations for turbulent flows". AIAA Paper No. 2001-1060.

[14] Han, D., and Hosder, S., 2014. "Inherent and epistemic uncertainty analysis for computational fluid dynamics simulations of synthetic jet actuators". Int. J. Uncertain. Quan., 4(6), pp. 511-533.

[15] Schaefer, J., Hosder, S., West, T., Rumsey, C., Carlson, J. R., and Kleb, W., 2016. "Uncertainty quantification of turbulence model closure coefficients for transonic wall-bounded flows". AIAA J., 55(1), pp. 195-213.

[16] Schaefer, J., Cary, A., Mani, M., Krakos, J., and Hosder, S., 2018. "Grid influence on turbulence model coefficient uncertainties in transonic wall-bounded flows". AIAA J., 56(8), pp. 3123-3137.

[17] Stephanopoulos, K., Whitte, I., Wray, T., and Agarwal, R. K., 2018. "Uncertainty quantification of turbulence model coefficients in OpenFOAM and Fluent for mildly separated flows". AIAA Paper No. 2018-3553.

[18] Oliver, T. A., and Moser, R. D., 2011. "Bayesian uncertainty quantification applied to RANS turbulence models". J. Phys. Conf. Ser., 318(4), p. 042032.

[19] Cheung, S. H., Oliver, T. A., Prudencio, E. E., Prudhomme, S., and Moser, R. D., 2011. "Bayesian uncertainty analysis with applications to turbulence modeling". Reliab. Eng. Syst. Safe., 96(9), pp. 1137-1149.

[20] Papadimitriou, D. I., and Papadimitriou, C., 2015. "Bayesian uncertainty quantification of turbulence models based on high-order adjoint". Comput. Fluids, 120, pp. 82-97.

[21] You, D., Wang, M., Moin, P., and Mittal, R., 2007. "Large-eddy simulation analysis of mechanisms for viscous losses in a turbomachinery tip-clearance flow". J. Fluid Mech., 586, pp. 177-204.

[22] Babinsky, H., and Harvey, J. K., 2011. Shock wave-boundary-layer interactions.

Cambridge

University Press, Cambridge, UK.

[23] Puterbaugh, S. L., and Copenhaver, W. W., 1997. "Flow field unsteadiness in the tip region of a transonic compressor rotor". ASME J. Fluids Eng., 119(1), pp. $122-128$.

[24] Gannon, A. J., and Hobson, G. V., 2009. "Pre-stall instability distribution over a transonic compressor rotor". ASME J. Fluids Eng., 131(5), p. 051106.

[25] Hah, C., and Shin, H. W., 2012. "Study of near-stall flow behavior in a modern transonic fan with compound sweep". ASME J. Fluids Eng., 134(7), p. 071101.

[26] Cumpsty, N. A., 1989. Compressor Aerodynamics. Longman Scientific and Technical, Harlow, UK.

[27] Driver, D. M., and Seegmiller, H. L., 1985. "Features of a reattaching turbulent shear layer in divergent channel flow". AIAA J., 23(2), pp. 163-171.

[28] Bachalo, W. D., and Johnson, D. A., 1986. "Transonic, turbulent boundary-layer separation generated on an axisymmetric flow model". AIAA J., 24(3), pp. 437-443.

[29] Sayma, A. I., Vahdati, M., Sbardella, L., and Imregun, M., 2000. "Modeling of three-dimensional viscous compressible turbomachinery flows using unstructured hybrid grids". AIAA J., 38(6), pp. 945-954.

[30] McKay, M. D., Beckman, R. J., and Conover, W. J., 1979. "Comparison of three methods for selecting values of input variables in the analysis of output from a computer code". Technometrics, 21(2), pp. 239-245.

[31] Nocedal, J., and Wright, S. J., 2006. Numerical Optimization. Springer, New York.

[32] Silverman, B. W., 1998. Density Estimation for Statistics and Data Analysis. Taylor \& Francis Group, New York. 